\title{
Spatial Correlation Based Clustering with Node Energy Based Multi-Hop Routing Scheme for Wireless Sensor Networks
}

\author{
Panchikattil Susheelkumar Sreedharan*, Dnyandeo Jageshwar Pete
}

\begin{abstract}
Major points of concern in implementing a wireless sensor network (WSN) are the network lifetime and energy utility within any delay tolerant network. Both these parameters define the success of the sensor network. The higher the expectancy of network Lifetime, the higher is the probability of acceptance of the network. Similarly, better the energy utilization in the network, better are the chances of success and implementation of the sensor network. Clustering is one such scheme adopted in WSN towards harnessing the best of above specified parameters for the network implemented. Most popular clustering techniques are the variants of LEACH protocol that facilitate cluster formation based on the proximity of an individual node to other nodes in the sensor network. These protocols are based on a single hop structure from the selected cluster heads in the network. This paper embarks on a multi-hop clustering algorithm that takes into consideration the spatial correlation between the nodes to form clusters and implements a highly energy efficient routing scheme which selects the multi-hop path in the network in a dynamic fashion.
\end{abstract}

Keywords: clustering; multi-hop; network lifetime; single-hop; spatial correlation

\section{INTRODUCTION}

Wireless Sensor Networks find its application in almost all fields of study like agriculture, environment monitoring, seismological study, defense and security and even extending into today's IoT [1]. It generally consists of high density sensor nodes that are spatially distributed in a given area of interest. Since these nodes are not easily humanly accessible, we have to be very particular about the energy utility in these nodes as its not possible to replace or recharge the batteries in them. Hence, many algorithms have adopted a clustering approach [2-11] with the cluster heads choosing a single hop data transfer mechanism to the sink. This mechanism is effective in case of small network area. But as the area of the sensor network grows in size, this particular mechanism can only support shorter network life time with reduced throughput. Standard algorithms like LEACH and its recent variants [4-15] are the examples of this particular approach. Here in this paper, we have proposed a cluster based multihop data transfer mechanism from the cluster head to the sink based on spatial correlation [16-23] between the scattered nodes. The routing path is arrived at dynamically based on the distance between the next possible hop satisfying a certain specified distance criterion and the minimum energy requirement to be satisfied by the next-hop cluster head. The performance of our proposed algorithm is compared with that of existing standard algorithm LEACH [24] and its recent variant Enhanced LEACH [7]. Therefore, here we have tried to implement an algorithm, which involves three phases .i.e. the clustering phase, cluster-head selection phase and the data transfer phase. The rest of the paper follows standard structure i.e. section II is dedicated to related work, section 3 $\& 4$ covers the models used while section 5 details the methodology involved. Section 6 covers the algorithms implemented, section 7 details the findings and results and lastly, section 8 concludes the findings of the paper.

\section{RELATED WORK}

Reference [3] introduces the standard clustering algorithm LEACH wherein the authors R. Sinde et al. (2020) uses a randomized way of selecting cluster head and also supports the data fusion or aggregation within the cluster. Here the operation during each round of data transfer is divided into two phases namely the set-up phase and the data transfer phase. During the setup phase, the cluster head is selected along with the cluster formation. In addition, during the data transfer phase, the sensed data flows from the cluster member nodes to their respective cluster head and the cluster heads aggregates the received data from all its members and transfers the aggregated data to the sink in a single hop. However, here the drawback lies in the fact that the cluster head is chosen in a randomized way not taking into consideration the residual energy of the candidate nodes for cluster head function. In addition, the effective communication path between the cluster member nodes and the sink through the cluster head is not considered for cluster formation process. These drawbacks have been taken into account and addressed to some extent in the recent variations of LEACH algorithm to some extent. In [13], the authors D. Mahmood et al. (2013) have altered the selection of cluster head in such a way that the cluster head continues to be the head till it's energy falls below a required threshold level. In addition, as far as data communication is concerned, they have envisaged intra cluster communication, inter cluster communication and cluster-head to sink communication and accordingly they have chosen two levels of amplification. The choice of these levels can be made dynamic thereby not fixing the number of levels at two. In [15], Zhidong et al. (2018) have devised a new strategy to arrive at the best optimum number of clusters balancing the energy need between the clusters as well as within each cluster. In their proposed scheme, they have introduced the distance variance factor in the initial set-up phase, and effectively implemented the node dormancy to save energy. Again, as the network 
area increases in size, the effectiveness of this algorithm also reduces since propagation of data involves two to three hops only. In [7], the authors Amer O. Abu Salem and Noor Shudifat (2019) have proposed an enhanced LEACH protocol, which takes into consideration the effective distance between the cluster members to the cluster head and from cluster head to the sink while implementing the cluster formation process. They have successfully shown that their scheme facilitates an extended network lifetime and reduced power usage in comparison to the standard protocol LEACH. But as the network area gets larger in size, even this protocol suffers due to higher levels of energy expended in transmitting the data to sinks which are very far located. Considering these shortcomings, our algorithms implements a multi hop mechanism for data propagation from the sensing nodes to the sink to have better energy efficiency and enhanced network lifetime.

\section{PROPOSED CORRELATION MODEL}

Sensing range or coverage area of any sensor node is a typical characteristic, which influences its region of sensing. Wireless Sensor networks are network of sensor nodes that typically consists of multiple nodes, which are more closely located. Since these nodes are closely located to each other in many applications, we can derive a spatial correlation between these nodes dictated by the overlapping region of coverage or the sensing region.

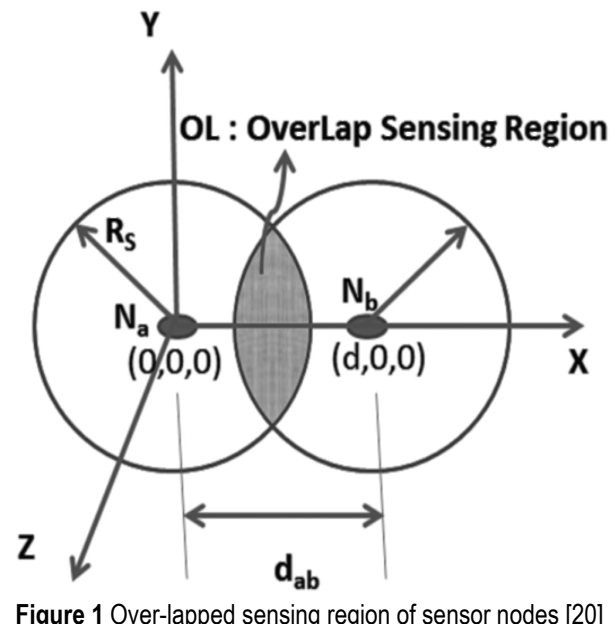

Fig. 1 represents two nodes that are closely located to each other, separated by a distance $d$ whose region of coverage is shown by the two circles with the nodes located at the Centre of each circle as depicted. We can observe that there is a region of overlap between the coverage areas of these two nodes that is highlighted by the shaded area. Now as the region of overlap increases the similarity in the data sensed by these nodes increases. Depending of the specific application requirement, we can have a trade-off between the data accuracy and the sensor nodes selected for communicating their sensed data instead of every node communicating their data to the sink. Hence, we try to make use of this spatial correlation between the sensor nodes in the development of our algorithm wherein clustering or cluster formation also takes into account the spatial correlation between these nodes.

Fig. 1 represents a two dimensional model of the sensor node, its coverage area and the region of overlap between their coverage region [25]. In real time the regions correlate to a three dimensional structure wherein each region represents a specific volume. Here, we define spatial correlation between two sensor nodes by the amount of overlap in their coverage area. We can say that, in a homogeneous sensor network, spatial correlation exists if the distance of separation between the two sensor nodes is less than twice the sensing radii of each node, which is quite evident from the study of geometry of the figure represented above. Moreover, as the distance between these two nodes increases beyond twice the sensing radii of the nodes, we say that there is no spatial correlation between these two nodes. In addition, the two nodes are $100 \%$ spatially correlated if they are co-located i.e. the distance of separation between them becomes zero. Considering these facts, we can define the overlap coefficient of correlation [29] between any two nodes $N_{\mathrm{a}}$ and $N_{\mathrm{b}}$ as:

$$
\begin{aligned}
\sigma_{\mathrm{ab}} & =\frac{\text { Volume of Overlap - of - Sensing Regions of Nodes } N_{\mathrm{a}}, N_{\mathrm{b}}}{\text { Combined Volume - of - Sensing Regions of Nodes } N_{\mathrm{a}}, N_{\mathrm{b}}} \\
\sigma_{\mathrm{ab}} & =\frac{V_{\mathrm{OL}}}{V_{\mathrm{Comb}}}
\end{aligned}
$$

where $V_{\mathrm{OL}}$ and $V_{\mathrm{Comb}}$ is the volume of the overlapping region of sensing of the two nodes and combined volume of sensing regions of the two nodes considered respectively.

We also define the region of overlap $(R O L)$ as:

$$
(\% R O L)_{\mathrm{ab}}=\sigma_{\mathrm{ab}} \times 100
$$

Using spherical geometry as given in [26], the numerator i.e. the dividend and denominator which is the divisor in Eq. (1) is expressed as:

$$
\begin{aligned}
& V_{\mathrm{OL}}=\frac{\pi}{12}\left(2 S R-d_{\mathrm{ab}}\right)^{2}\left(d_{\mathrm{ab}}-4 S R\right) \\
& V_{\mathrm{Comb}}=\frac{8 \pi \times S R^{3}}{3}-\frac{\pi}{12}\left(2 S R-d_{\mathrm{ab}}\right)^{2}\left(d_{\mathrm{ab}}+4 S R\right)
\end{aligned}
$$

Combining (1), (3) and (4), we express the overlap coefficient of correlation between the two nodes as:

$$
\sigma_{\mathrm{ab}}=\frac{\left(2 S R-d_{\mathrm{ab}}\right)^{2}\left(d_{\mathrm{ab}}+4 S R\right)}{32 S R^{3}-\left(2 S R-d_{\mathrm{ab}}\right)^{2}\left(d_{\mathrm{ab}}+4 S R\right)}
$$

where $S R$ is assumed to be the uniform node sensing range, $N_{\mathrm{a}}, N_{\mathrm{b}}$ are the two nodes considered at a distance dab from each other.

Thus, it can be expressed that $\sigma_{\mathrm{ab}}=0$ for any two nodes separated by a distance greater than twice the sensing range 
of each node i.e. $2 S R$. Hence, we can sum-up the $S R$ correlation coefficient between two nodes separated by a distance $\mathrm{d}$ as:

$$
\sigma=\left\{\begin{array}{ccc}
\frac{(2 S R-d)^{2}(d+4 S R)}{32 S R^{3}-(2 S R-d)^{2}(d+4 S R)} & \text { if } & 0 \leq d<2 S R \\
0 & \text { if } & d \geq 2 S R
\end{array}\right\}
$$

Expression (6) represents the sensing region correlation model [20].

\section{ENERGY MODEL}

The standard reference energy model is taken from ref. [9] which gives a detailed analysis of two main types of energy associated with WSN namely the propagation energy which is the energy involved in the transmission of data from the sensor nodes to the sink and energy used for various electronics involved before transmission after reception of data in the nodes. The propagation distance decides whether the propagation energy is influenced by free space propagation model or multi-path propagation model. If the propagation distance is less than the crossover distance it is the free space propagation that defines the propagation energy and if the propagation distance is beyond the crossover distance, the propagation energy is defined by the multi space propagation model as given in ref. [9, 26, 27]. Using this model, the energy involved in the transmission of 1-bit message is expressed as:

$E_{\mathrm{T}}=l \times E_{\mathrm{EX}}+l \times p_{1} \times d_{\mathrm{T}}^{(n)}$

where $E_{\mathrm{EX}}$ is the energy/bit involved in the electronics, $l \times p_{1} \times d_{\mathrm{T}}^{(n)}$ is the propagation-energy for 1-bit message for covering a transmission distance $d$ with a propagation loss exponent $n$.

Ref. [26] states for any transmission distance less than the crossover distance $d_{0}$, the free space model is applied and the expression for the transmission energy is given as:

$$
E_{\mathrm{T} 1}=l \times E_{\mathrm{EX}}+l \times \varepsilon_{\mathrm{fs}} \times d_{\mathrm{T}}^{(2)}
$$

where $\varepsilon_{\mathrm{fs}}=p_{\mathrm{l}}$, i.e. the propagation loss for free space.

And for any transmission distance greater than the crossover distance $d_{0}$, the multi-path model is applied and the transmission energy is stated as:

$$
E_{\mathrm{T} 2}=l \times E_{\mathrm{EX}}+l \times \varepsilon_{\mathrm{mp}} \times d_{\mathrm{T}}^{(4)}
$$

where $\varepsilon_{\mathrm{mp}}=p_{\text {l }}$, i.e. the propagation loss in multi-path transmission. Here cross-over distance $\mathrm{d}_{0}$ is given by:

$$
d_{0}=\sqrt{\frac{\varepsilon_{\mathrm{fs}}}{\varepsilon_{\mathrm{mp}}}}
$$

\section{PROPOSED METHOD}

Here we have selected a wireless sensor network area of 200 by $200 \mathrm{~m}^{2}$ with a dense spread of sensor nodes in the given area. The total number of nodes are 1000 that are initially given a random spread across the network. The initial random locations of all these nodes are maintained the same throughout the simulation for varying parameters and algorithms under study in this paper. The sink is assumed to be centrally located and aware of all the GPS enabled node's location detail. The network is supposed to be a static homogeneous network that is the nodes are stationary in nature and uniformly energized at the beginning of implementation. All the nodes transmits their location detail to the sink and the sink implements the centralized clustering algorithm to form cluster groups based on the spatial correlation criterion chosen which is maintained throughout the network lifetime. The cluster information is relayed by sink to all the nodes which is now aware of its cluster details that is member ids and member locations. All the nodes keep a record of its neighboring nodes detail that are separated by a maximum specified distance (of $5 S R$ ) from itself which is the limitation placed for next hop node. Thus cluster formation phase is only implemented once in the lifetime of network. After the cluster formation phase, then we implement a cluster head election phase through a distributed algorithm implemented at the individual nodes in the network. This algorithm estimates a fitness function value for the node based on the leftover energy after the previous rounds of data transfer, its distance from sink and the sum of propagation distance from other member nodes to the candidate $\mathrm{CH}$ node within the cluster. Each node relays this value to its other cluster members and the node with largest valued fitness function is chosen as the cluster head for the cluster, thus presenting a dynamic $\mathrm{CH}$ selection process. The $\mathrm{CHs}$ then relay their election as $\mathrm{CH}$ to other member nodes as well as other $\mathrm{CHs}$ located nearby which are in the range of $5 S R$ from itself. Initially all the $\mathrm{CHs}$ are by-default the main cluster heads. Few amongst these cluster heads that satisfy the defined minimum spatial correlation factor of 0.1 with their neighboring cluster head and whose fitness value is lower than neighbor cluster head are labeled as secondary cluster head. Now, here all the cluster members that satisfy a minimum spatial correlation factor of 0.1 with its cluster head are made to sleep, thereby avoiding any redundant data as defined in the application requirement based on the tradeoff chosen between data accuracy and number of active sensing nodes transmitting their sensed data i.e. energy conservation. All the members of associated secondary cluster are also checked for the satisfaction of minimum spatial correlation factor with the associated main cluster head too and are either made dormant or live depending on the conditions prevalent. Immediately after the segregation of the cluster heads as main or secondary cluster heads, each of the main cluster head finds the next hop node (which is also a designated main cluster head) to chart out the route forward towards the sink. These next hop nodes are selected only if they satisfy the minimum and maximum propagation distance fixed for being eligible which is between $2 S R$ and $5 S R$. All these 
intermediate routing nodes performs the role of a repeater for other main cluster head's relayed packets. In the absence of finding a suitable intermediate routing node, the cluster heads are programmed to directly send the message to the sink. Speaking of the data flow in the network, the secondary cluster heads will remove the redundant data received from its cluster members and forwards the aggregated data to the associated main cluster head for further transmission. The main cluster head also aggregates the data received from its cluster members and the associated secondary cluster heads and relays the aggregated data either to the sink directly or to the next hop cluster head node as estimated by the distributed algorithm running at the cluster head node itself. The sinks keeps a record of all the dead nodes or renders any node as dead if it does not receive any information from that node in the aggregated packet message relayed by its main cluster head in three consecutive rounds of data transfer. The sink is aware of the dead nodes and therefore has an estimate of the total number of packets to be received from the network. On receiving messages from all the active clusters or waiting for pre-specified buffer-time, it relays the start of next round to all nodes in the network. All the cluster heads on receiving the round-start message from the sink drops the data if any received by it and pending for further relay and starts initial phase of estimating the fitness function value for the next round under consideration, further followed up by the subsequent stages as defined in the implementing distributed algorithm at the node. The entire implementation is simulated using MATLAB@2016a wherein the cluster formation is carried out for various values of Spatial correlation factor ranging from 0.1 to 1 (one). Here we have chosen five values of spatial correlation factor i.e. $0.1,0.2,0.3,0.5$ and 0.7 for simulation purpose and studied the effect of the variation in spatial correlation coefficient on the network lifetime and throughput in our proposed approach along with the comparison of each results (specific values of correlation coefficient) with standard Algorithm namely LEACH and its recent variant called Enhanced LEACH.

\section{ALGORITHM}

The proposed scheme is implemented using a combination of centralized algorithm run at the sink and a distributed algorithm run at each node. The centralized algorithm is taken from paper $[28,29]$ that facilitates the cluster formation at the sink side and then relaying the cluster information to the sensor nodes in the wireless sensor network area. The distributed algorithm accomplishes three tasks namely the cluster head selection and designating them as main cluster head or secondary cluster head followed by the next hop node estimation and finally the data transfer phase from the sensing node to the sink. The centralized cluster forming algorithm is shown in the flowchart in Fig. 2:

The cluster formation process is followed by the distributed algorithm that accomplishes the three tasks mentioned earlier namely the cluster head selection and designating them as main cluster head or secondary cluster head followed by the next hop node estimation and finally the data transfer phase. The distributed algorithm implemented at the node level is depicted in the flow chart given in Fig. 3. The fitness-function ( $f f$ ) value is expressed using the following expression $[28,29]$ :

$$
f f=\left(\frac{W_{\mathrm{a}} \times N_{\mathrm{LOE}}+W_{\mathrm{b}}\left(\frac{1}{S P D^{2}}\right)}{N_{\mathrm{LOE}}+\left(\frac{1}{S P D^{2}}\right)}\right)
$$

where $f f$ represents fitness-function value for the node supporting its candidature for the role of $\mathrm{CH}$, $\mathrm{LOE}$ represents its left-over energy (residual energy) after the previous round, $W_{\mathrm{a}}$ and $W_{\mathrm{b}}$ are the proportional weights expressed as:

$$
W_{\mathrm{a}}=\left(\frac{D_{\mathrm{NS}}}{D_{\mathrm{NS}}+S P D}\right), W_{\mathrm{b}}=\left(\frac{S P D}{D_{\mathrm{NS}}+S P D}\right)
$$

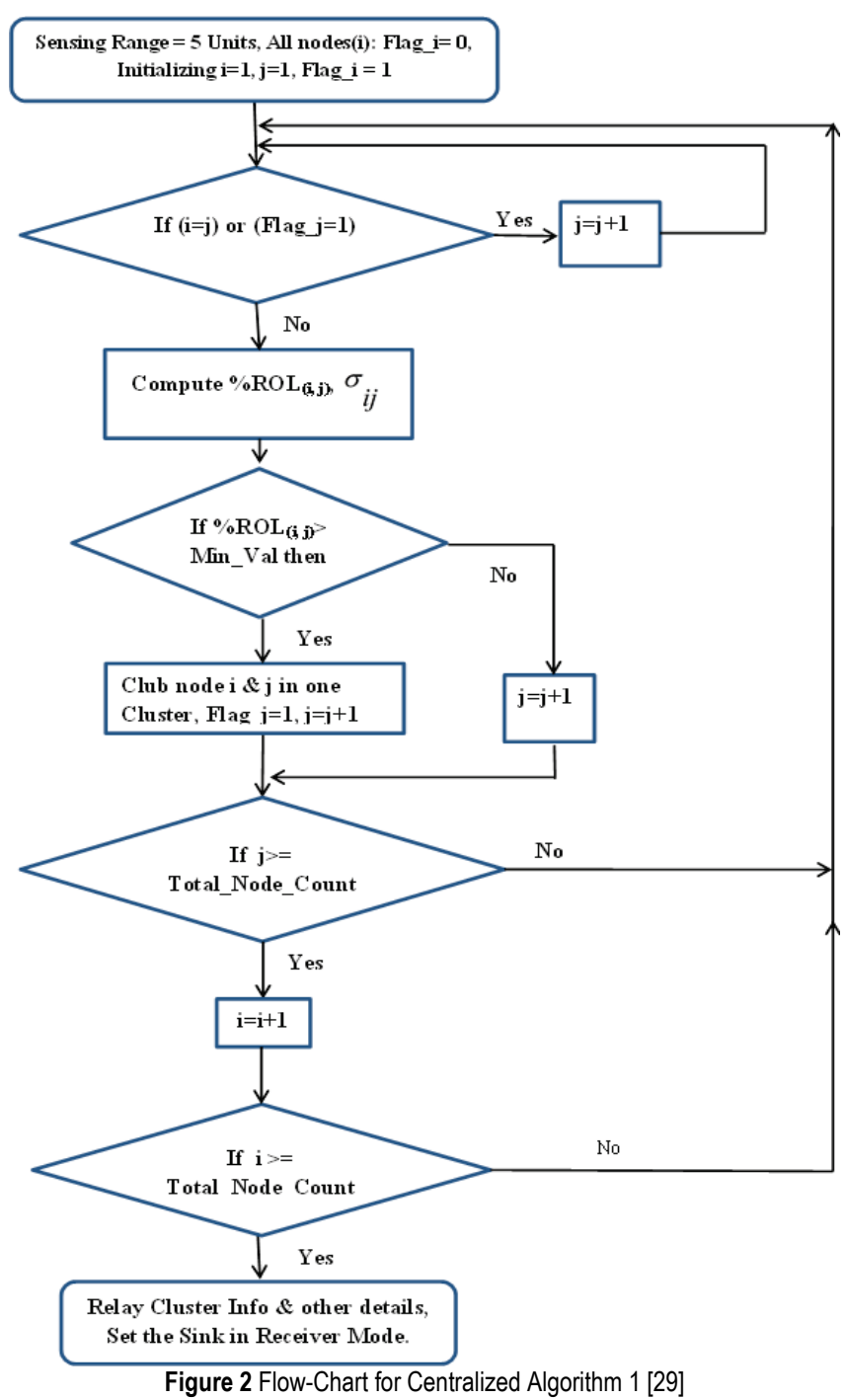

Here $D_{\mathrm{NS}}$ represents distance between the node and sink, $S P D$ represents sum of propagation distance. $S P D$ is the total propagation distance encountered in a cluster owing to the 


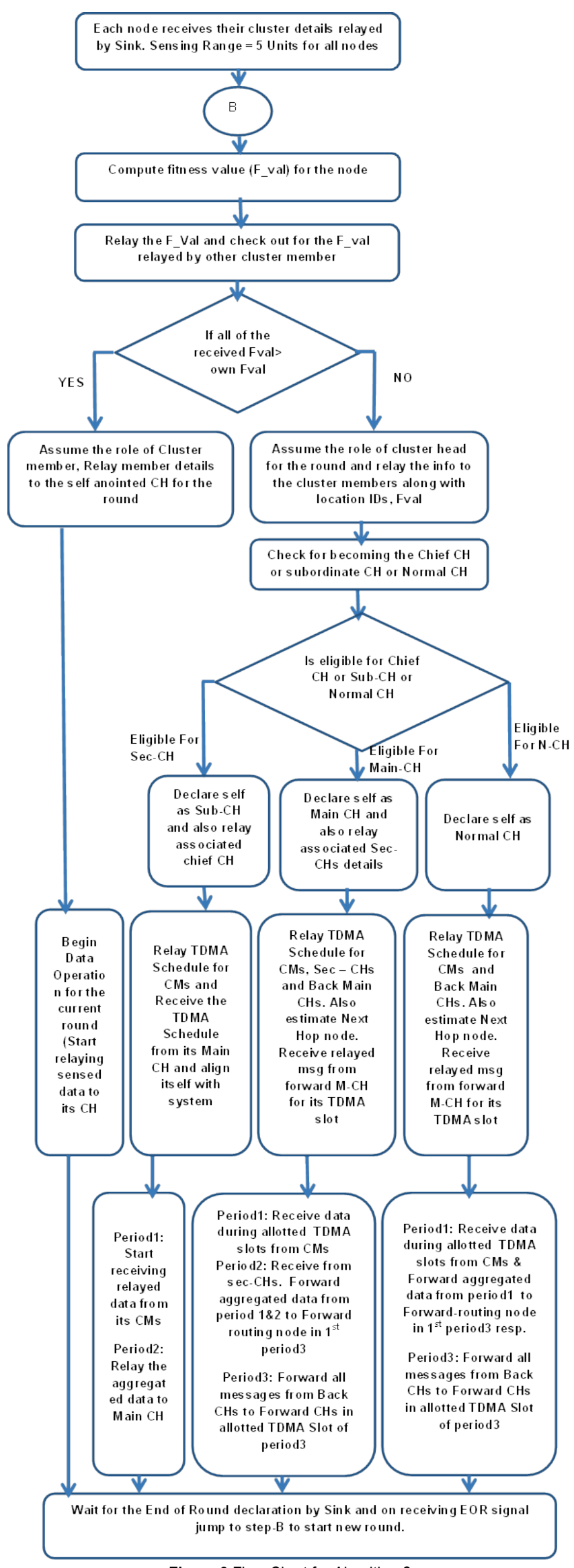

Figure 3 Flow-Chart for Algorithm 2 relay of messages from the cluster member to the candidate cluster head in the cluster for the round under consideration. $W_{\mathrm{a}}$ and $W_{\mathrm{b}}$ are dynamic proportional weights used to estimate the fitness function of the candidate node for cluster head election taking into consideration both the LOE of the node and the expendable energy in the cluster by the member nodes for intra-cluster communication (which should be minimum) proportionately. If $S P D$ is maximum, that means more energy needs to be expended cumulatively by the cluster members and if SPD is minimum less energy needs to be expended cumulatively by the cluster members. Hence, ideally when $S P D$ is lower, $f f$ value should be higher and when $S P D$ is higher, $f f$ value should be lower which is satisfied by Eq. (10). Using the fitness function value, the distributed algorithm enables the selection of main cluster heads, secondary cluster heads and the further routing paths for the round under consideration. Each cluster head maintains a TDMA schedule for each of its cluster members for message transfers. The first period of each round is solely dedicated for cluster members to relay their data to their cluster heads. The second period is for secondary cluster heads to relay their data to the main cluster heads using a TDMA schedule dictated by its main cluster head. The third period is for the movement of aggregated data from the main cluster heads to their first forward routing node. A TDMA Schedule is also maintained by the forward (towards sink) main cluster head for all its neighboring back main cluster head (which are further away from the sink) that are in the range of $5 S R$. The third period is repeated till sink signals the end of round. Thus, using the defined TDMA schedules the data flow is simulated from the sensing nodes to the sink through various intermediary routing nodes. The sink receives the forwarded messages from various clusters and regularly checks for the reception of messages from all the active clusters. If the sink has received the aggregated data message from all the active clusters, it signals the end of round otherwise it waits for a specific period of time which is more than sufficient for the message from the farthest cluster to reach the sink before it signals the end of round to all the cluster members.

\section{RESULT}

In the simulation, we have enforced the above parameter values as specified in Tab. 1 . We have implemented the simulation with varying degrees of correlation value chosen for clustering. Here the correlation value is varied between 0 and 100 percent and typically for our simulation we have selected a correlation value of $0.7,0.5,0.3,0.2$, and 0.1 (i.e. $70 \%, 50 \%, 30 \%, 20 \%$ and $10 \%$ ) as the sample correlation values for which we have implemented our multi-hop routing algorithm. Under each correlation value, we have three instances of simulation with a minimum node energy parameter value set to each node at $10 E_{\mathrm{min}}, 500 E_{\mathrm{min}}$, 1000 Emin respectively to support multi-hop routing of data. Here $E_{\min }$ is taken as 0.0002 Joules and hence $10 E_{\min }, 500 E_{\min }$ and $1000 E_{\min }$ which corresponds to a minimum nodal residual energy of 0.002 Joules, 0.1 Joules and 0.2 Joules respectively set for each node to participate in multi-hop 
routing during each of the three instances of simulation respectively. The results for the various instances of simulations are represented below graphically. The results are expressed in terms of network energy utilization, throughput for the various algorithms under consideration and the network life-time reflected for all the algorithms under study. The network life-time is assumed to be the round of data transfer supported by the respective algorithm till the death of $70 \%$ of the sensing nodes present in the network. And the maximum throughput of the networks is taken as the total number of message packets arriving at the sink during the life-time of network. For the purpose of comparison in regards with the network energy consumption, we have taken the reference of LEACH and EnhancedLEACH algorithm's network lifetime and it is observed that the network energy is depleted in case of LEACH and enhanced-LEACH before data transfer round number 1500 . The simulation results encompasses the results for the standard algorithm LEACH, its advanced version EnhancedLEACH and three instances of our proposed algorithm with varying values of correlation fixed for Spatial correlation based clustering.

Table 1 Parameters taken for simulation (@MATLAB 2016a)

\begin{tabular}{|c|l|c|}
\hline No & \multicolumn{1}{|c|}{ Design Para-meters } & Value/ Symbol \\
\hline 1 & Total Sensor Nodes & 1000 \\
\hline 2 & WSN Area & $200 \times 200 \mathrm{~m}^{2}$ \\
\hline 3 & Initial energy of all sensor nodes & $0.5 \mathrm{~J}$ \\
\hline 4 & Sensing Range (SR) of Node: & $5 \mathrm{~m}$ \\
\hline 5 & $\begin{array}{l}\text { Free-Space factor for propagation distance } \\
\text { less than cross-over distance } d_{0}\left(d<d_{0}\right)\end{array}$ & $10 \mathrm{~nJ} / \mathrm{bit} / \mathrm{m}^{2}$ \\
\hline 6 & Multi path factor for longer distance $\left(d>d_{0}\right)$ & $0.0013 \mathrm{pJ} / \mathrm{bit} / \mathrm{m}^{4}$ \\
\hline 7 & $\begin{array}{l}\text { Energy required for reception } \& \text { transmission } \\
\text { of signals by Electronics involved }\end{array}$ & $50 \mathrm{~nJ} / \mathrm{bit}$ \\
\hline 8 & Energy expended for data aggregation & $5 \mathrm{~nJ} / \mathrm{bit}$ \\
\hline 9 & No of bits per message packet & $4000 \mathrm{bits}$ \\
\hline
\end{tabular}

The simulation for a correlation value of $\sigma=0.7$ is depicted below with the three instances for the three minimum nodal energy parameter value set in our proposed algorithm. The graphical results of the three instances of simulation for our proposed algorithm with the set parameter values along with the simulation results for existing standard LEACH and Enhanced LEACH protocol is given below in Fig. 4(a), Fig. 4(b) and Fig. 4(c).

Fig. 4(a) represents the Network's energy utilization curve with respect to the rounds of data transfer rounds supported by the network for standard algorithms LEACH and Enhanced-LEACH in comparison to the three instances of our proposed algorithm with $\sigma=0.7$.

Fig. 4(b) represents the Network's throughput curve with respect to the rounds of data transfer supported for all the existing protocols and instances of our proposed algorithm with $\sigma=0.7$ while Fig. 4(c) represents the comparative network lifetime with respect to the rounds of data transfer supported for all the existing protocols and instances of our proposed algorithm with $\sigma=0.7$.

Similarly, we have implemented the simulation for varying values of correlation and the graphical results for the same are presented below. For correlation value of $\sigma=0.5$, we have the following results.

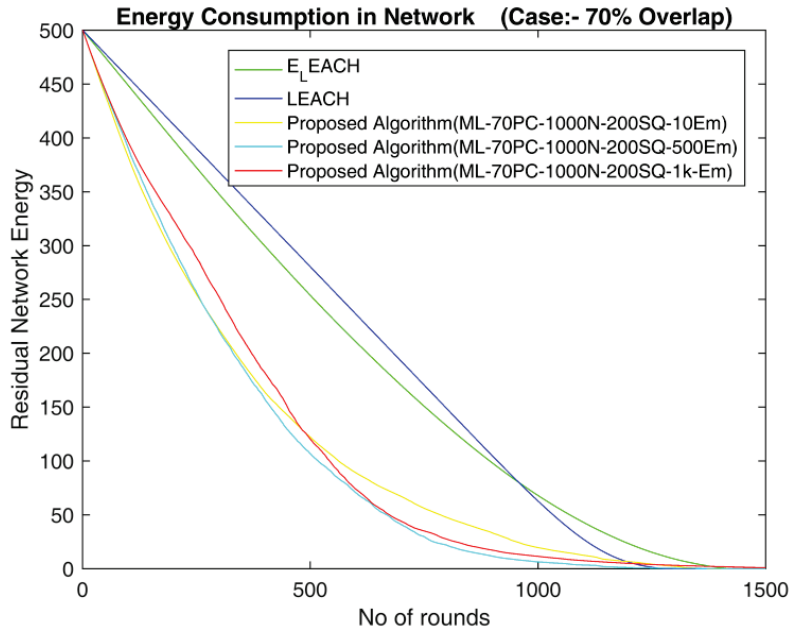

Figure 4(a) Network Energy Utilization for LEACH, Enhanced LEACH and three instances of our proposed Algorithm $(\sigma=0.7)$

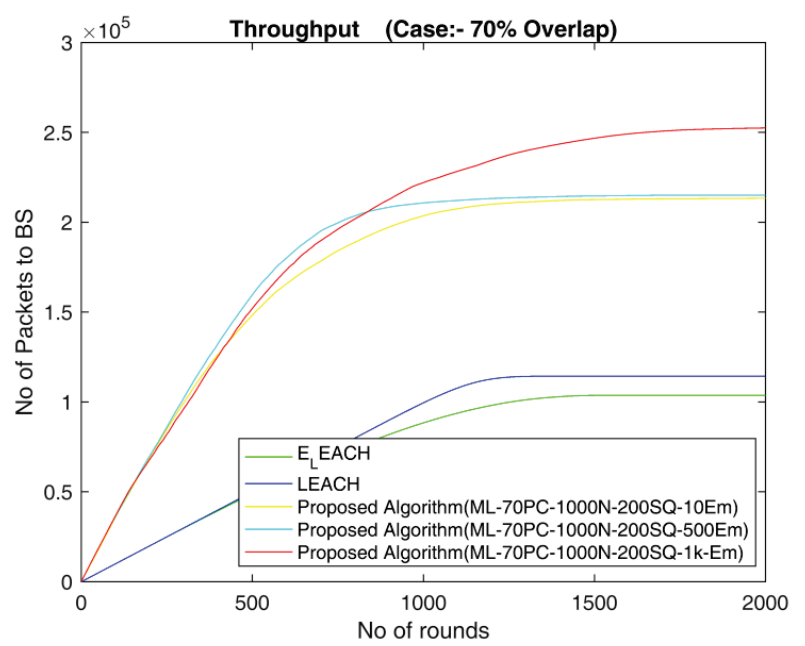

Figure 4(b) Network's Throughput curve for LEACH, Enhanced LEACH and three instances of our proposed Algorithm $(\sigma=0.7)$

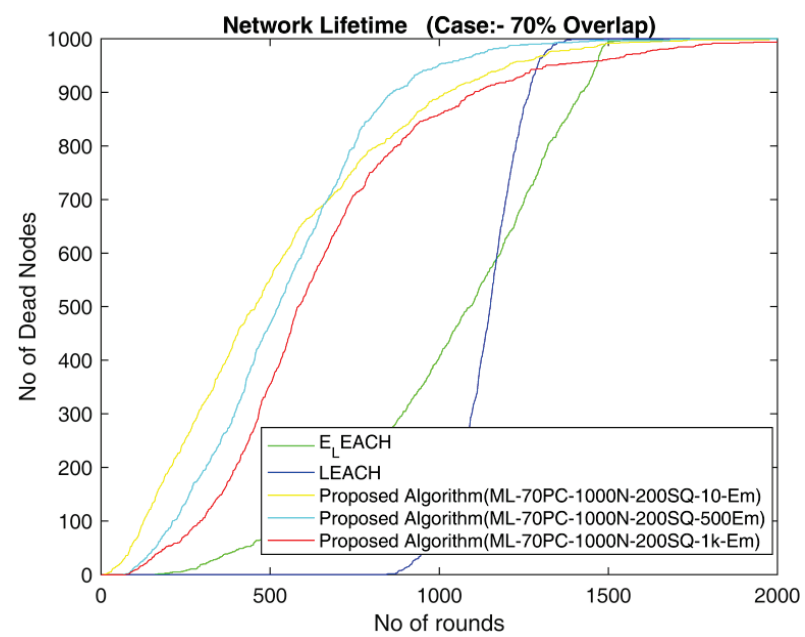

Figure 4(c) Network Lifetime for LEACH, Enhanced LEACH and three instances of our proposed Algorithm $(\sigma=0.7)$

Fig. 5(a) represents the Network's energy utilization curve with respect to the rounds of data transfer rounds supported by the network for standard algorithms LEACH 
and Enhanced-LEACH in comparison to the three instances of our proposed algorithm with $\sigma=0.5$.

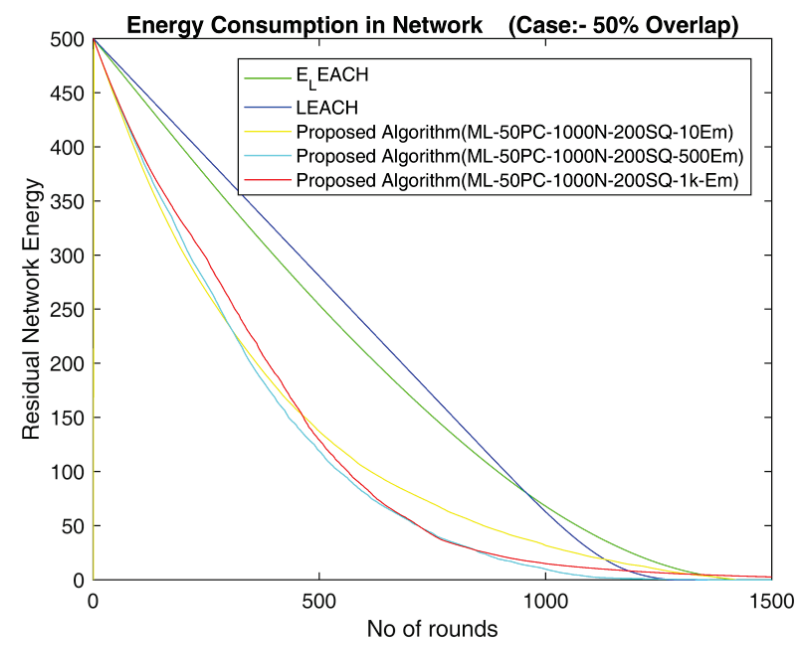

Figure 5(a) Network Energy Utilization for LEACH, Enhanced LEACH and three instances of our proposed Algorithm $(\sigma=0.5)$

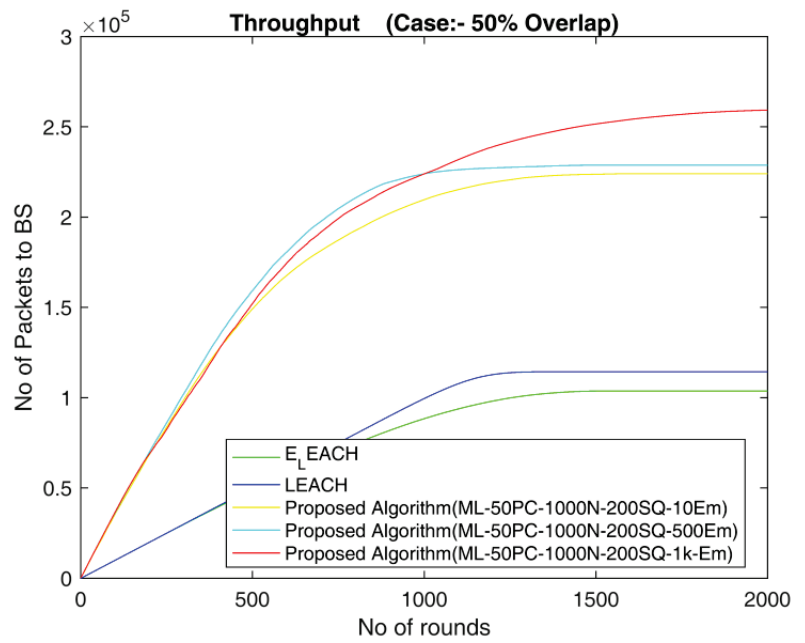

Figure 5(b) Network's Throughput curve for LEACH, Enhanced LEACH and three instances of our proposed Algorithm $(\sigma=0.5)$

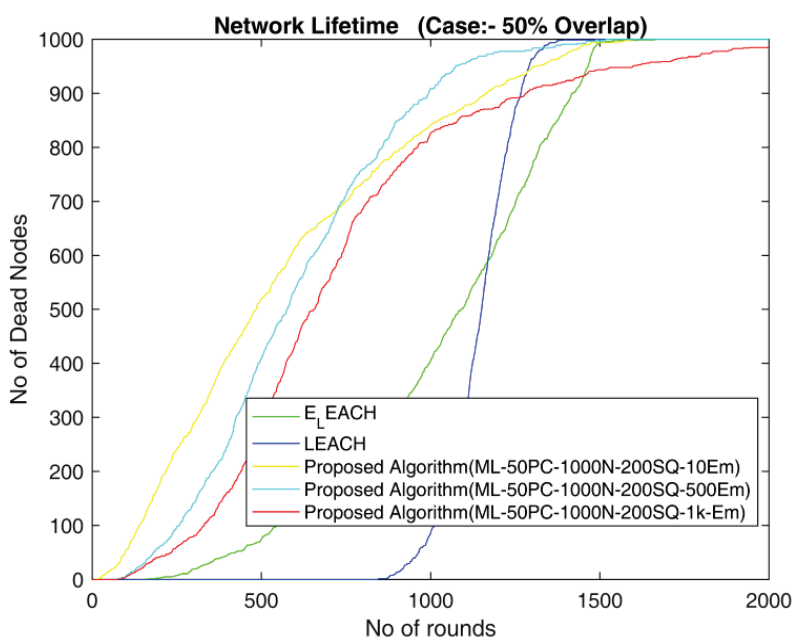

Figure 5(c) Network Lifetime for LEACH, Enhanced LEACH and three instances of our proposed Algorithm $(\sigma=0.5)$

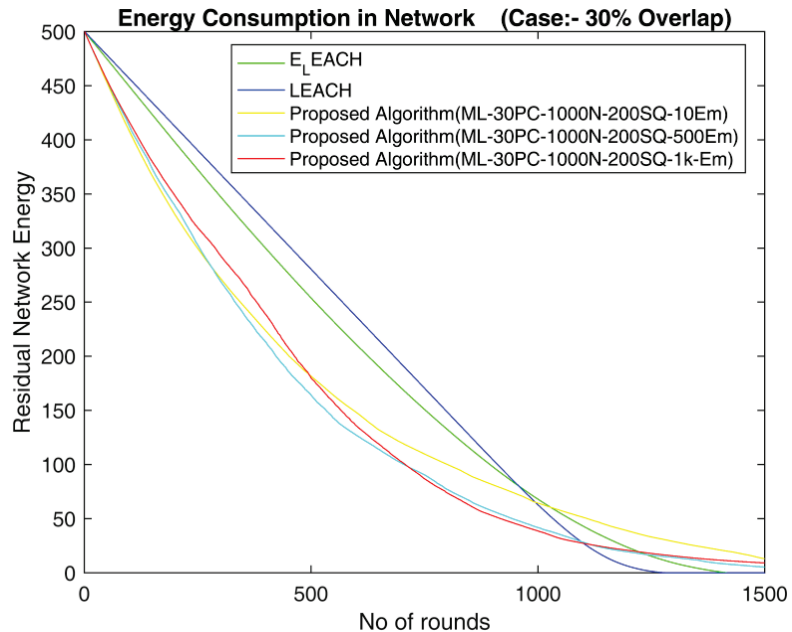

Figure 6(a) Network Energy Utilization for LEACH, Enhanced LEACH and three instances of our proposed Algorithm $(\sigma=0.3)$

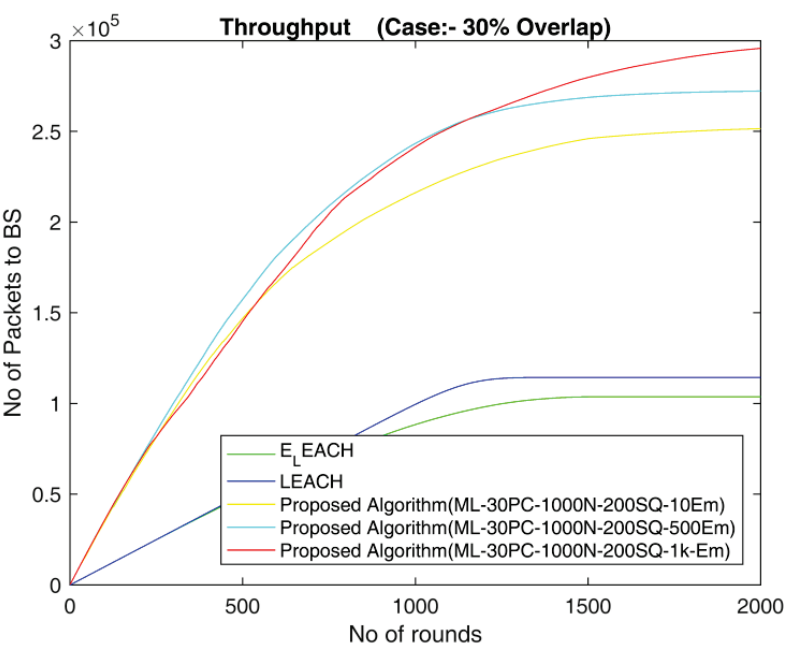

Figure 6(b) Network's Throughput curve for LEACH, Enhanced LEACH and three instances of our proposed Algorithm $(\sigma=0.3)$

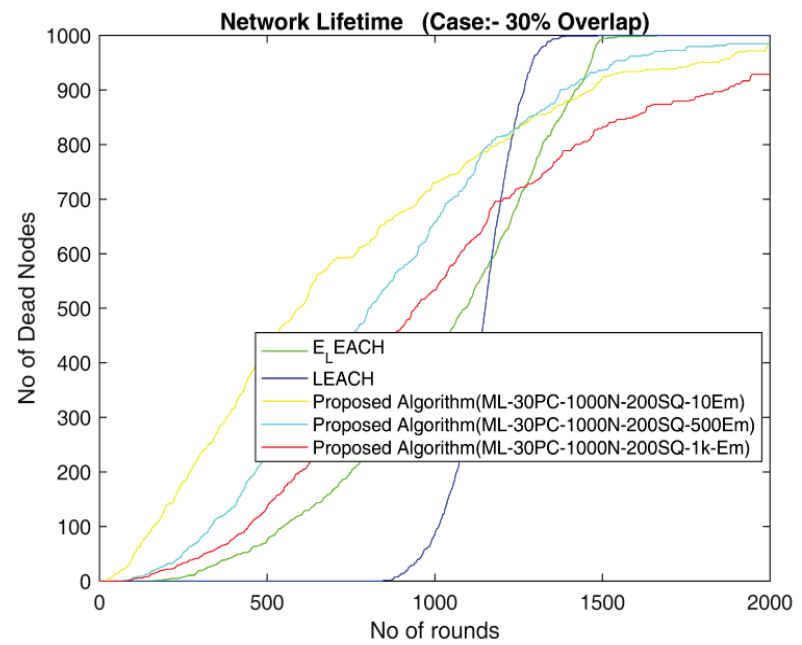

Figure 6(c) Network Lifetime for LEACH, Enhanced LEACH and three instances of our proposed Algorithm $(\sigma=0.3)$

Fig. 5(b) represents the Network's throughput curve with respect to the rounds of data transfer supported for all the existing protocols and instances of our proposed algorithm 
with $\sigma=0.5$ while Fig. 5(c) represents the comparative network lifetime with respect to the rounds of data transfer supported for all the existing protocols and instances of our proposed algorithm with $\sigma=0.5$.

For correlation value of $\sigma=0.3$, we have the following results:

Fig. 6(a) represents the Network's energy utilization curve with respect to the rounds of data transfer rounds supported by the network for standard algorithms LEACH and Enhanced-LEACH in comparison to the three instances of our proposed algorithm with $\sigma=0.3$.

Fig. 6(b) represents the Network's throughput curve with respect to the rounds of data transfer supported for all the existing protocols and instances of our proposed algorithm with $\sigma=0.3$ while Fig. 6(c) represents the comparative network lifetime with respect to the rounds of data transfer supported for all the existing protocols and instances of our proposed algorithm with $\sigma=0.3$.

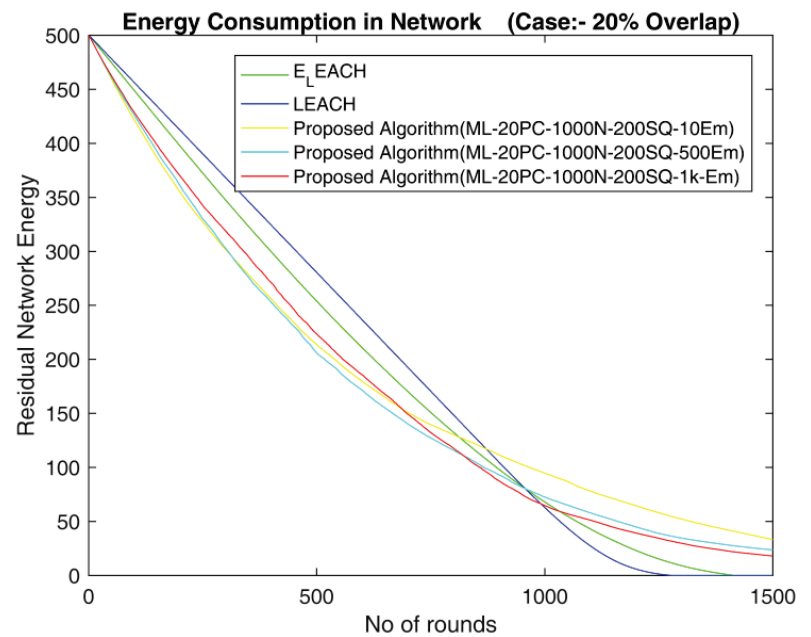

Figure 7(a) Network Energy Utilization for LEACH, Enhanced LEACH and three instances of our proposed Algorithm $(\sigma=0.2)$

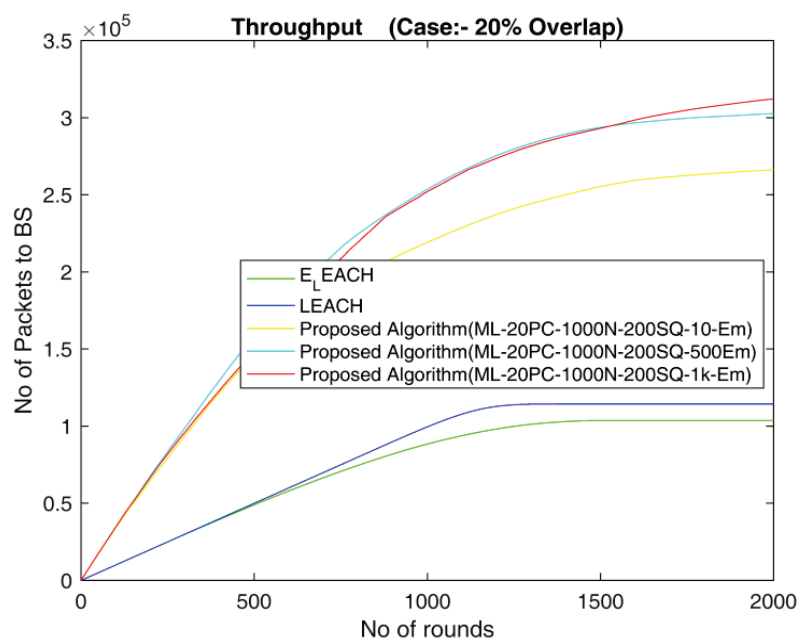

Figure 7(b) Network's Throughput curve for LEACH, Enhanced LEACH and three instances of our proposed Algorithm $(\sigma=0.2)$

For correlation value of $\sigma=0.2$, we have the following results:

Fig. 7(a) represents the Network's energy utilization curve with respect to the rounds of data transfer rounds supported by the network for standard algorithms LEACH and Enhanced-LEACH in comparison to the three instances of our proposed algorithm with $\sigma=0.2$.

Fig. 7(b) represents the Network's throughput curve with respect to the rounds of data transfer supported for all the existing protocols and instances of our proposed algorithm with $\sigma=0.2$ while Fig. 7(c) represents the comparative network lifetime with respect to the rounds of data transfer supported for all the existing protocols and instances of our proposed algorithm with $\sigma=0.2$.

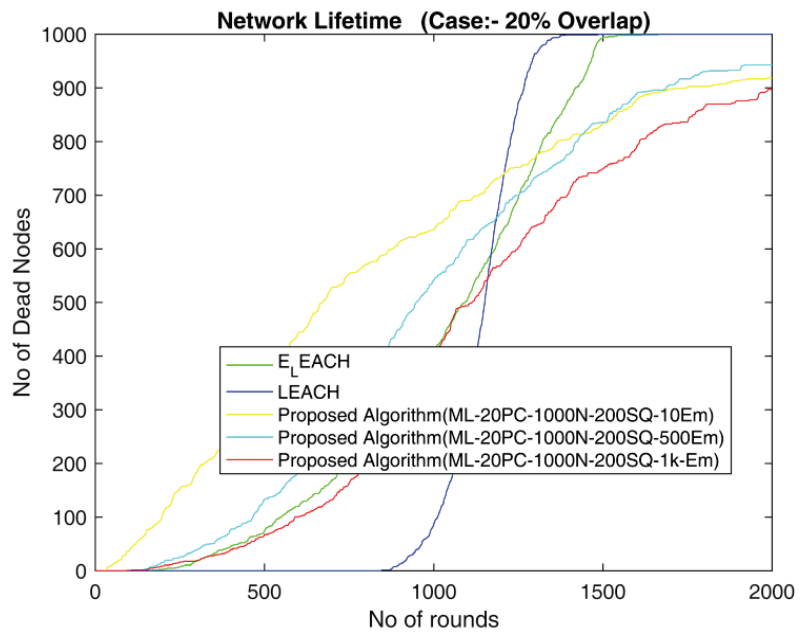

Figure 7(c) Network Lifetime for LEACH, Enhanced LEACH and three instances of our proposed Algorithm $(\sigma=0.2)$

For correlation value of $\sigma=0.1$, we have the following results:

Fig. 8(a) represents the Network's energy utilization curve with respect to the rounds of data transfer rounds supported by the network for standard algorithms LEACH and Enhanced-LEACH in comparison to the three instances of our proposed algorithm with $\sigma=0.1$.

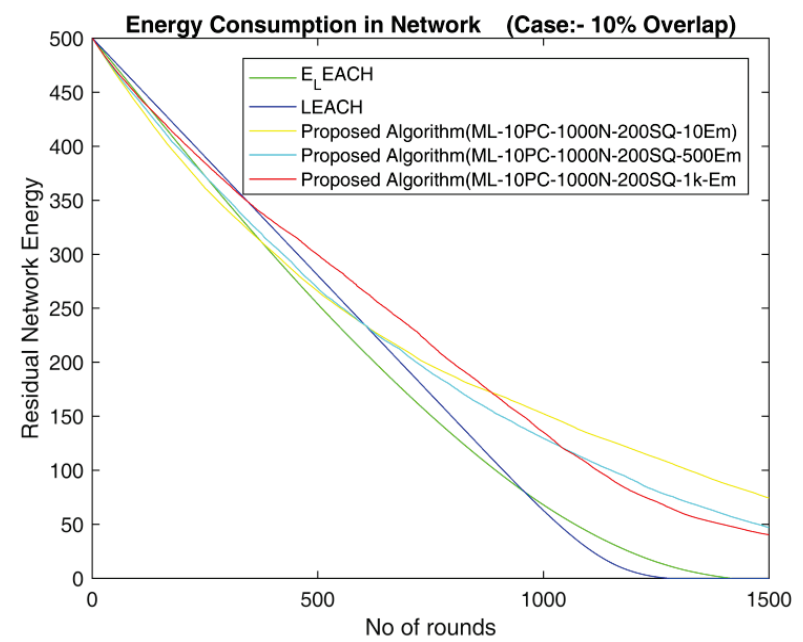

Figure 8(a) Network Energy Utilization for LEACH, Enhanced LEACH and three instances of our proposed Algorithm $(\sigma=0.1)$

Fig. 8(b) represents the Network's throughput curve with respect to the rounds of data transfer supported for all the 
existing protocols and instances of our proposed algorithm with $\sigma=0.1$ while Fig. 8(c) represents the comparative network lifetime with respect to the rounds of data transfer supported for all the existing protocols and instances of our proposed algorithm with $\sigma=0.1$.

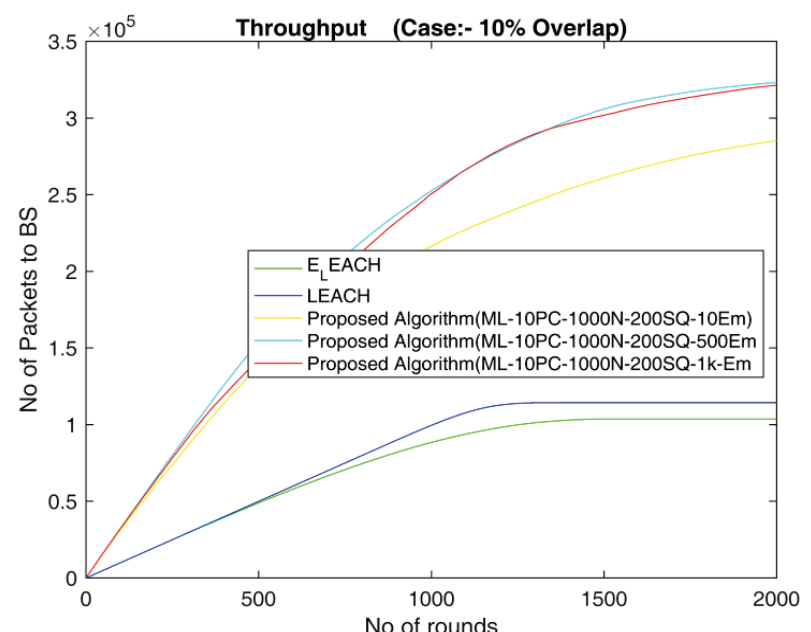

Figure 8(b) Network's Throughput curve for LEACH, Enhanced LEACH and three instances of our proposed Algorithm $(\sigma=0.1)$

The above graphical results are represented in tabular form in Tab. 2 and Tab. 3. Tab. 2 represents the output values corresponding to the results obtained during the simulation of existing algorithms standard LEACH and EnhancedLEACH. Tab. 3 on the other hand represents the various output values of the various instances during the simulation of our proposed algorithm with $\sigma=0.7,0.5,0.3,0.2$ and 0.1 .

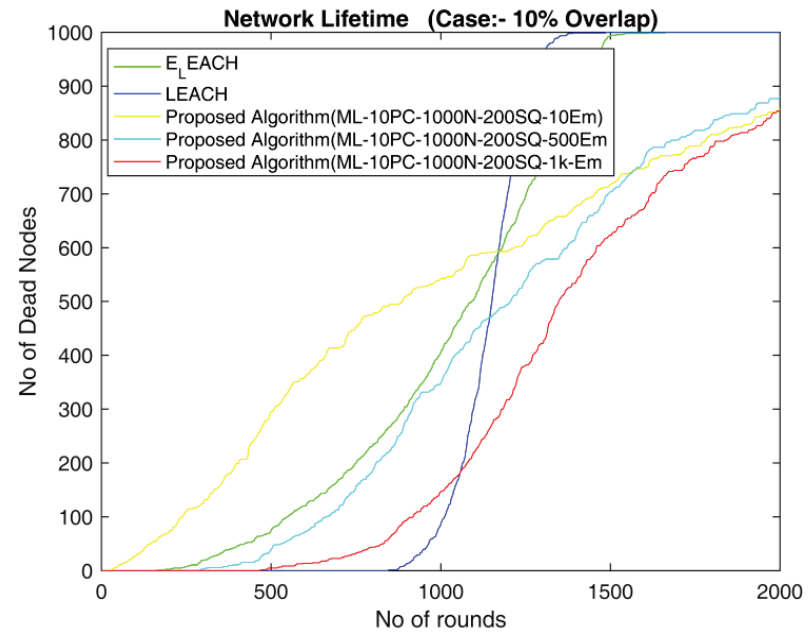

Figure 8(c) Network Lifetime for LEACH, Enhanced LEACH and three instances of our proposed Algorithm $(\sigma=0.1)$

Table 2 Parameters observed during simulation of LEACH, ENHANCED LEACH algorithms

\begin{tabular}{|l|c|c|}
\hline \multirow{2}{*}{\multicolumn{1}{|c|}{ Parameters/ Algorithms }} & \multicolumn{2}{c|}{$\begin{array}{r}100-\text { Sensor Nodes in } \\
200 \times 200 \mathrm{~m}^{2}\end{array}$} \\
\cline { 2 - 3 } & LEACH & E-LEACH \\
\hline Clusters formed Roundwise between: & 1 to 128 & 1 to 134 \\
\hline 1st Node Death (ND) Round & 845 & 160 \\
\hline 10\% ND Round & 1011 & 546 \\
\hline 20\% ND Round & 1065 & 750 \\
\hline $50 \%$ ND Round & 1152 & 1091 \\
\hline $70 \%$ ND Round (LifeTime) & 1198 & 1252 \\
\hline Throughput & 112795 & 100009 \\
\hline Balance Network Energy after 1500 rounds & 0 & 0 \\
\hline
\end{tabular}

Table $3 \sigma$ and corresponding results for 1000 Nodes spread in a WSN Area of $200 \times 200 \mathrm{~m}^{2}$

\begin{tabular}{|c|c|c|c|c|c|c|c|c|c|c|c|c|c|c|c|}
\hline$\sigma$ & \multicolumn{3}{|c|}{0.7} & \multicolumn{3}{|c|}{0.5} & \multicolumn{3}{|c|}{0.3} & \multicolumn{3}{|c|}{0.2} & \multicolumn{3}{|c|}{0.1} \\
\hline $\begin{array}{c}E_{\min } \text { Set for } \\
\text { Forwarding } \\
\text { Agent }\end{array}$ & $\begin{array}{c}E_{\min }= \\
0.002 \mathrm{~J}\end{array}$ & $\begin{array}{c}E_{\min }= \\
0.1 \mathrm{~J}\end{array}$ & $\begin{array}{c}E_{\min }= \\
0.2 \mathrm{~J}\end{array}$ & $\begin{array}{c}E_{\min }= \\
0.002 \mathrm{~J}\end{array}$ & $\begin{array}{c}E_{\min }= \\
0.1 \mathrm{~J}\end{array}$ & $\begin{array}{c}E_{\min }= \\
0.2 \mathrm{~J}\end{array}$ & $\begin{array}{c}E_{\min }= \\
0.002 \mathrm{~J}\end{array}$ & $\begin{array}{c}E_{\min }= \\
0.1 \mathrm{~J}\end{array}$ & $\begin{array}{c}E_{\min }= \\
0.2 \mathrm{~J}\end{array}$ & $\begin{array}{c}E_{\min }= \\
0.002 \mathrm{~J}\end{array}$ & $\begin{array}{c}E_{\min }= \\
0.1 \mathrm{~J}\end{array}$ & $\begin{array}{c}E_{\min }= \\
0.2 \mathrm{~J}\end{array}$ & $\begin{array}{c}E_{\min }= \\
0.002 \mathrm{~J}\end{array}$ & $\begin{array}{c}E_{\min }= \\
0.1 \mathrm{~J}\end{array}$ & $\begin{array}{c}E_{\min }= \\
0.2 \mathrm{~J}\end{array}$ \\
\hline $\begin{array}{c}\text { No of } \\
\text { Clusters } \\
\text { present }\end{array}$ & 953 & 953 & 953 & 847 & 847 & 847 & 634 & 634 & 634 & 522 & 522 & 522 & 374 & 374 & 374 \\
\hline $\begin{array}{l}1 \text { st ND } \\
\text { Round }\end{array}$ & 9 & 68 & 74 & 9 & 74 & 74 & 21 & 64 & 74 & 27 & 93 & 93 & 23 & 175 & 465 \\
\hline $\begin{array}{c}20 \% \mathrm{ND} \\
\text { Round }\end{array}$ & 205 & 311 & 399 & 217 & 354 & 439 & 269 & 466 & 596 & 321 & 627 & 798 & 404 & 810 & 1077 \\
\hline $\begin{array}{c}50 \% \text { ND } \\
\text { Round }\end{array}$ & 457 & 524 & 582 & 484 & 576 & 656 & 595 & 803 & 941 & 671 & 948 & 1113 & 885 & 1205 & 1348 \\
\hline $\begin{array}{c}70 \% \text { ND } \\
\text { Round }\end{array}$ & 674 & 670 & 740 & 750 & 737 & 816 & 952 & 1050 & 1205 & 1130 & 1241 & 1395 & 1454 & 1493 & 1621 \\
\hline $\begin{array}{c}\text { Throughput } \\
(\mathrm{K})\end{array}$ & 175.48 & 191.02 & 194.92 & 187.30 & 202.94 & 207.06 & 211.74 & 248.28 & 260.44 & 231.42 & 278.97 & 287.72 & 257.82 & 305.42 & 308.29 \\
\hline
\end{tabular}
below:

The step-wise analysis of the above results is given

Here we have taken the life-time of networks as the time or the round till the number of active sensor nodes in the network falls below $30 \%$ of the actual nodes present at the beginning. In other words, it is the time or round till which the number of dead nodes touches or surpasses $70 \%$ of the total number of nodes present in the network.

\subsection{Observation-1}

From Tab. 2, it is clear that standard LEACH protocol supports 1198 rounds of data transfer with a throughput of 
1,12,795 packets while Enhanced-LEACH supports 1252 rounds of data transfer with a throughput of $1,00,009$. Thus we observe that Enhanced-LEACH gives a better network lifetime in comparison to Standard LEACH protocol but its throughput is reduced in comparison to Enhanced-LEACH protocol.

\subsection{Observation-2}

From Tab. 3, it is observed that for $\sigma=0.7$ and $\sigma=0.5$, our algorithm is not at all competitive with either LEACH or Standard LEACH is concerned as far as network-lifetime is concerned. But as you decrease the value of $\sigma$ to 0.2 and 0.1 , we observe that when we increase the minimum residual energy required for all the sensor nodes to participate in the data routing process as a forwarding agent, there is a measurable improvement in the network lifetime which is better than that observed with standard LEACH and Enhanced LEACH. For the simulation of our proposed algorithm with $\sigma=0.2$ and the minimum node residual energy of $0.1 \mathrm{~J}$, the network lifetime observed is 1241 almost equal to that observed in LEACH with 1252 rounds of data transfer. But when minimum node residual energy $\left(E_{\min }\right)$ is fixed at $0.2 \mathrm{~J}$, the network lifetime in our proposed algorithm increases drastically to 1395 rounds of data transfer.

Similarly the simulation of our algorithm with $\sigma=0.1$ with various values of minimum residual energy set for each node to be allowed to participate in the data routing process shows much better network lifetime than that observed with LEACH and Enhanced LEACH simulation. The network lifetime for our proposed algorithm with $\sigma=0.1$ and a minimum data-routing-participating residual energy for nodes set at $0.002 \mathrm{~J}, 0.1 \mathrm{~J}$ and $0.2 \mathrm{~J}$ is at 1454,1493 and 1621 respectively which reflects a drastic improvement over the measurements seen in LEACH and Enhanced LEACH.

\subsection{Observation-3}

As far as throughput measurements are concerned, it is reflected from Tab. 2 and Tab. 3 that throughput in our proposed algorithm increases from 1,75,487 packets to a maximum of 3,08,290 packets for correlation value of $\sigma=0.7$ to $\sigma=0.1$ which are comparatively much higher values than that observed with the simulation of LEACH and Enhanced LEACH each with a throughput value of $1,12,795$ and $1,00,009$ respectively.

\subsection{Observation-4}

As far as the energy utility parameter is concerned, it is seen from the graphs in Fig. 4(a) and Fig. 5(a) that for $\sigma=0.7$ and $\sigma=0.5$, energy consumption in LEACH and Enhanced LEACH is better than all the instances of proposed Algorithm. It is observed that the energy in the network is exhausted before round number 750 in all instances of simulation using our proposed algorithm with $\sigma=0.7$ and $\sigma$ $=0.5$ which is approximately half the Network-Lifetime observed in LEACH and Enhanced LEACH.

But as the value of $\sigma$ is further decreased, we observe a gradual improvement in Network Lifetime using our proposed algorithm over LEACH and Enhanced LEACH. It is observed that for $\sigma=0.3,0.2$ and 0.1 even after round number 1500 , which is taken as a reference, there is network energy balance in the network and the amount of network energy balance in the network increases as the value of $\sigma$ decreases from 0.3 to 0.1 , thereby increasing the networklifetime.

For $\sigma=0.2$ and $\sigma=0.1$, we observe the best performance for our proposed algorithm with reference to the performance of LEACH and Enhanced LEACH, wherein even after 1500 rounds of data transfer, there is a network energy balance ranging from $18 \mathrm{~J}$ to $74 \mathrm{~J}$ respectively for the various instances considered for $\sigma$. This improvement in network energy consumption is reflected in the enhanced lifetime of the network using our proposed algorithm having $\sigma=0.1$ with a network lifetime of 1454 rounds, 1493 rounds, 1621 rounds for the three instances of our algorithm defined by $E_{\min }=0.002,0.1$ and 0.2 respectively which is much higher than the network lifetime seen in LEACH and Enhanced LEACH. Similarly for $\sigma=0.2$, the improvement in network energy consumption is seen from the observed data represented in Tab. 2 and Tab. 3. In this case of our proposed algorithm we arrive at a network lifetime of 1130 rounds, 1241 rounds, 1395 rounds associated with the three instances of our algorithm defined by $E_{\min }=0.002,0.1$ and 0.2 respectively. Thus the observed lifetime in this case with $E_{\min }$ $=0.2$ shows drastic improvement over LEACH and Enhanced LEACH.

\section{CONCLUSION}

From the above findings presented in section 7 , we come to the following conclusions:

For $\sigma=0.7$ and 0.5 , the results achieved are inferior to standard LEACH and Enhanced LEACH algorithms as far as network lifetime is concerned. But throughput is better using our proposed algorithm which is greater than LEACH and Enhanced LEACH by about 1.2 to 1.4 times.

But as we decrease the value of $\sigma$ to 0.3 , we see throughput increases from 1.8 to 2.3 times the throughput observed using LEACH and Enhanced LEACH. But the network Lifetime lags behind LEACH and Enhanced LEACH very narrowly.

Further as we decrease the value of $\sigma$ to 0.2 , we can conclude from the findings and results that the throughput is enhanced to around 2.05 to 2.5 times the throughput observed in LEACH and Enhanced LEACH. As far as the network lifetime is concerned, the lifetime is almost comparable to that achieved in Enhanced LEACH in case of second instance $\left(E_{\min }=0.1 \mathrm{~J}\right)$ of our Proposed Algorithm. Similarly for the third instance $\left(E_{\min }=0.2 \mathrm{~J}\right)$ of our proposed algorithm, the network lifetime surpasses the lifetime as seen with Enhanced LEACH by around 150 rounds.

Lastly when the value of $\sigma$ is decreased to 0.1 , from the findings and results of the previous section we can conclude that the throughput using our proposed algorithm is enhanced to around 2.28 to 2.73 times the throughput available with LEACH and Enhanced LEACH. And as far as the network 
lifetime is concerned, our proposed algorithm gives a better network lifetime which shows a rise of around 200 to 350 additional rounds of data transfer in comparison to standard algorithms LEACH and Enhanced LEACH which converges to better energy utilization in our proposed algorithm.

\section{Notice}

This paper was presented at IC2ST-2021 - International Conference on Convergence of Smart Technologies. This conference was organized in Pune, India by Aspire Research Foundation, January 9-10, 2021. The paper will not be published anywhere else.

\section{REFERENCES}

[1] Sohraby, K., Minoli, D., \& Znati, T. (2007). Wireless Sensor Networks: Technology, Protocols and Applications. John Wiley and Sons, Hoboken, New Jersey, 38-71. https://doi.org/10.1002/047011276X

[2] Taqieddin, E., Awad, F., \& Ahmad, H. (2017). Location-Aware and Mobility-Based Performance Optimization for WSN. Journal of Wireless Mobile Networks, Ubiquitous Computing, \& Dependable Applications, 8(3), 38-59.

[3] Sinde, R., Begum, F., Njau, K., \& Kaijage, S. (2020). Refining Network Lifetime of WSN using Energy-Efficient Clustering and DRL-based Sleep Scheduling. Sensors, 20(5), 1540. https://doi.org/10.3390/s20051540

[4] Li, Z. \& Xin, P. (2017). Evidence-Efficient Multihop Clustering Routing Scheme for Large-Scale Wireless Sensor Networks. Wireless Communications and Mobile Computing, Article-ID: 1914956. https://doi.org/10.1155/2017/1914956

[5] Ebadi, S. (2012). A Multihop Clustering Algorithm for Energy Saving in Wireless Sensor Networks. International Scholarly Research Network - ISRN Sensor Networks. https://doi.org/10.5402/2012/817895

[6] Izadi, D., Abawajy, J., \& Ghanavati, S. (2015). An Alternative Clustering Scheme in WSN. IEEE Sens J, 15(7), 4148-4155. https://doi.org/10.1109/JSEN.2015.2411598

[7] Amer, O., Salem, A., \& Noor, S. (2019). Enhanced LEACH Protocol for Increasing a Lifetime of WSNs. Personal and Ubiquitous Computing, 23, 901-907. https://doi.org/10.1007/s00779-019-01205-4

[8] Arora, V., Sharma, V., \& Sachdeva, M. (2016). A Survey on LEACH and other Routing Protocols in Wireless Sensor Network. Optik, 127(16), 6590-6600. https://doi.org/10.1016/j.ijleo.2016.04.041

[9] Comeau, F. \& Aslam, N. (2011). Analysis of LEACH Energy Parameters. Workshop on Emerging Topics in Sensor Networks (EmSeNs). Procedia Computer Science, 5, 933-938. https://doi.org/10.1016/j.procs.2011.07.131

[10] Tamilarasi, N. \& Parvathi, K. (2016). Extension of Network Lifetime using Fuzzy C-Means, Model and Cluster Hierarchy Concept. International Journal of Recent Trends in Engineering \& Research, 2(8), 302-308. https://doi.org/10.5121/ijdps.2012.3505

[11] Rathi, N., Saraswat, J., \& Bhattacharya, P. (2012). A Review on Routing Protocols for Application in Wireless Sensor Networks. International Journal of Distributed and Parallel Systems, 3(5). https://doi.org/10.5121/ijdps.2012.3505

[12] Xu, L., Collier, R., \& O'Hare, G. (2017). A Survey of Clustering Techniques in WSNs and Consideration of the
Challenges of Applying such to $5 \mathrm{G}$ IoT Scenarios. IEEE Internet of Things Journal, 4(5), 1229-1249. https://doi.org/10.1109/JIOT.2017.2726014

[13] Mahmood, D., Javaid, N., Mahmood, S., Qureshi, S., Memon, A., \& Zaman, T. (2013). MODLEACH: A Variant of LEACH for WSNs. Eighth International Conference on Broadband and Wireless Computing, Communication and Applications, Compiegne, 158-163. https://doi.org/10.1109/BWCCA.2013.34

[14] Bhavana, T. \& Murthy, J. (2014). Spatial Correlation based Clustering-Algorithm for Random \& Uniform Topology in WSNs. IJRET: International Journal of Research in Engineering and Technology, 3(6), 83-87. https://doi.org/10.15623/ijret.2014.0306015

[15] Zhao, Z., Xu, K., Hui, G., \& Hu, L. (2018). An EnergyEfficient Clustering Routing Protocol for Wireless Sensor Networks based on AGNES with Balanced Energy Consumption Optimization. Sensors, 18(11), 3938. https://doi.org/10.3390/s18113938

[16] Vuran, M., Akan, Ö., \& Akyildiz, I. (2004). Spatiotemporal Correlation: Theory \& Applications for WSNs. Comput Networks, 45(3), 246-258. https://doi.org/10.1016/j.comnet.2004.03.007

[17] Tsai, M. \& Huang, T. (2014). A Sub-Clustering Algorithm based on Spatial Data Correlation for Energy Conservation in Wireless Sensor Networks. Sensors, 14(11), 21858-21871. https://doi.org/10.3390/s141121858

[18] Liu, Z., Xing, W., Zeng, B., Wang, Y., \& Lu, D. (2013). Distributed Spatial Correlation-based Clustering for Approximate Data Collection in WSNs. IEEE $27^{\text {th }}$ Int Conf on Adv Information Networking and Applications. https://doi.org/10.1109/AINA.2013.26

[19] Jorio, A., Fkihi, S., Elbhiri, B., \& Aboutajdine, D. (2015). An Energy-Efficient Clustering Routing Algorithm based on Geographic Position and Residual Energy for WSN. J Comput Networks Commun. https://doi.org/10.1155/2015/170138

[20] Panchikattil, S. S. \& Pete, D. J. (2020). Spatial Clustering with Sequential CH Selection for Energy-Efficient WSN. In: Vasudevan H., Gajic Z., Deshmukh A. (eds) Proceedings of International Conference on Wireless Communication. Lecture Notes on Data Engineering and Communications Technologies, 36. Springer, Singapore. https://doi.org/10.1007/978-981-15-1002-1_30

[21] Wang, X., Liu, X., Wang, M., Nie, Y., \& Bian, Y. (2019). Spatial Query-Centric Geographic Routing Protocol in Wireless Sensor Network. Sensors, 19(10), 2363. https://doi.org/10.3390/s19102363

[22] Zhou, Y., Yang, L., \& Ni, M. (2019). Novel Energy-Efficient Data Gathering Scheme Exploiting Spatial-Temporal Correlation for Wireless Sensor Networks. Wireless Communications and Mobile Computing. https://doi.org/10.1155/2019/4182563

[23] Pourpeighambar, S. \& Sabaei M. (2013). Spatial Correlation Aware Protocols for Efficient Data Aggregation of Moving Object in Wireless Sensor Networks. Scientia Iranica, 20(3), 695-709. https://doi.org/10.1016/j.scient.2012.11.010

[24] Heinzelman, W., Chandrakasan, A., \& Balakrishnan, H. (2000). Energy-Efficient Communication Protocol for Wireless Microsensor Networks. Proceedings of the $33^{\text {rd }}$ Annual Hawaii International Conference on System Sciences, 2, Maui, HI, USA, pp. 10. https://doi.org/10.1109/HICSS.2000.926982

[25] Khalid, Z. \& Durrani, S. (2013). Distance Distributions in Regular Polygons. IEEE Transactions on Vehicular Technology, 62(5), 2363-2368. https://doi.org/10.1109/TVT.2013.2241092 
[26] Kole, S., Vhatkar, K., \& Bag, V. (2014). Distance based Cluster Formation Technique for LEACH Protocol in Wireless Sensor Network. International Journal of Application or Innovation in Engineering \& Management (IJAIEM), 3(3), 334-338.

[27] Heinzelman, W., Chandrakasan, A., \& Balakrishnan, H. (2002). An Application-Specific Protocol Architecture for Wireless Microsensor Networks. IEEE Transactions on Wireless Communications, 1(4), 660-670. https://doi.org/10.1109/TWC.2002.804190

[28] Sreedharan, P. \& Pete, D. (2020). Dynamic Multi Hop Spatial Clustering Scheme for Data Transfer in Wireless Sensor Networks with Enhanced QoS M. 2020 International Conference for Emerging Technology (INCET), Belgaum, India, 1-8. https://doi.org/10.1109/INCET49848.2020.9154118

[29] Sreedharan, P. S. \& Pete, D. J. (2020). Spatial Clustering Algorithm with Dynamic Multi Hop Routing for Wireless Sensor Networks. International Journal on Emerging Technologies, 11(3), 1169-1178.

\section{Authors' contacts:}

Panchikattil Susheelkumar Sreedharan, Research Scholar Dept. of Electronics Engg., Datta Meghe College of Engineering, Sector-3, Airoli, Navi Mumbai-400 708, Maharashtra, India E-mail: susheelkumarsredh34@gmail.com

Dnyandeo Jageshwar Pete, Professor \& Head

Dept. of Electronics Engg., Datta Meghe College of Engineering, Sector-3, Airoli, Navi Mumbai-400 708, Maharashtra, India

E-mail: pethedj@rediffmail.com 